\title{
Fekete-Szegö problem for generalized bi-subordinate functions of complex order
}

\author{
Sercan Kazımoğlu (D), Erhan Deniz*(D) \\ Department of Mathematics, Faculty of Science and Letters, Kafkas University, Campus, 36100, \\ Kars-Turkey
}

\begin{abstract}
In this paper, we obtain the Fekete-Szegö inequality for the generalized bi-subordinate functions of complex order. The various results, which are presented in this paper, would generalize those in related works of several earlier authors.
\end{abstract}

Mathematics Subject Classification (2010). 30C45, 30C80

Keywords. analytic functions, starlike functions, convex functions, Ma-Minda starlike functions, Ma-Minda convex functions, subordination, Fekete-Szegö inequality

\section{Introduction}

Let $\mathcal{A}$ be the class of analytic functions in the open unit disk $\mathbb{D}=\{z \in \mathbb{C}:|z|<1\}$ and let $\mathcal{S}$ be the class of functions $f$ that are analytic, univalent in $\mathbb{D}$ and are of the form

$$
f(z)=z+\sum_{k=2}^{\infty} a_{k} z^{k}
$$

The Koebe one-quarter theorem assures that the image of unit disk $\mathbb{D}$ under every univalent function $f \in \mathcal{A}$ contains a disk of radius $1 / 4$. Thus every univalent function $f$ has an inverse $f^{-1}$ satisfying

$$
f^{-1}(f(z))=z(z \in \mathbb{D}) \text { and } f\left(f^{-1}(w)\right)=w, \quad\left(|w|<r_{0}, r_{0} \geq 1 / 4\right) .
$$

Furthermore, the Taylor-Maclaurin series of $f^{-1}$ is given by

$$
f^{-1}(w)=w-a_{2} w^{2}+\left(2 a_{2}^{2}-a_{3}\right) w^{3}-\cdots .
$$

A function $f \in \mathcal{A}$ is said to be bi-univalent in $\mathbb{D}$ if $f$ is univalent and $f^{-1}$ has univalent analytic continuation, which we denote by $g$, to the unit disk $\mathbb{D}$. Let $\sigma$ denote the class of bi-univalent functions defined in the unit disk $\mathbb{D}$. Coefficient problem for bi-univalent functions were recently investigated by several authors $[1,4-8,15-17,19,20]$. A function $f \in$ $\mathcal{A}$ is said to be subordinate to a function $h \in \mathcal{A}$, denoted by $f \prec h$, if there exists an analytic function $w \in \mathcal{B}_{0}$, where $\mathcal{B}_{0}:=\{w: w(0)=0,|w(z)|<1, \quad z \in \mathbb{D}\}$ such that $f(z)=$ $h(w(z))$. We let $\mathcal{S}^{*}$ consist of starlike functions $f \in \mathcal{A}$, that is, $\operatorname{Re}\left\{z f^{\prime}(z) / f(z)\right\}>0$ in $\mathbb{D}$ and $\mathcal{C}$ consist of convex functions $f \in \mathcal{A}$, that is, $1+\operatorname{Re}\left\{z f^{\prime \prime}(z) / f^{\prime}(z)\right\}>0$ in $\mathbb{D}$. Ma and

\footnotetext{
*Corresponding Author.

Email addresses: srcnkzmglu@gmail.com (S. Kazımoğlu), edeniz36@gmail.com (E. Deniz)

Received: 22.04.2019; Accepted: 13.12.2019
} 
Minda [12] unified various subclasses of starlike and convex functions for which either of the quantity $z f^{\prime}(z) / f(z)$ or $1+z f^{\prime \prime}(z) / f^{\prime}(z)$ is subordinate to a more general superordinate function. For this purpose, they considered an analytic function $\varphi$ with positive real part in the unit disk $\mathbb{D}$ and normalized by $\varphi(0)=1$ and $\varphi^{\prime}(0)>0$. The class of Ma-Minda starlike functions consists of functions $f \in \mathcal{A}$ satisfying the subordination $z f^{\prime}(z) / f(z) \prec \varphi(z)$. Similarly, the class of Ma-Minda convex functions consists of functions $f \in \mathcal{A}$ satisfying the subordination $1+z f^{\prime \prime}(z) / f^{\prime}(z) \prec \varphi(z)$. Extensions of the above two classes (see [14]) are

and

$$
\mathcal{S}^{*}(\gamma ; \varphi) \equiv\left\{f \in \mathcal{A}: 1+\frac{1}{\gamma}\left(\frac{z f^{\prime}(z)}{f(z)}-1\right) \prec \varphi(z), \quad \gamma \in \mathbb{C} \backslash\{0\}\right\}
$$

$$
C(\gamma ; \varphi) \equiv\left\{f \in \mathcal{A}: 1+\frac{1}{\gamma}\left(\frac{z f^{\prime \prime}(z)}{f^{\prime}(z)}\right) \prec \varphi(z), \quad \gamma \in \mathbb{C} \backslash\{0\}\right\} .
$$

In literature, the functions belonging to these classes are called Ma-Minda starlike and convex of complex order $\gamma(\gamma \in \mathbb{C} \backslash\{0\})$, respectively. A function $f$ is bi-starlike of MaMinda type of complex order $\gamma(\gamma \in \mathbb{C} \backslash\{0\})$ and bi-convex of Ma-Minda type of complex order $\gamma(\gamma \in \mathbb{C} \backslash\{0\})$ if both $f$ and $g$ are ,respectively, Ma-Minda starlike and convex of complex order $\gamma(\gamma \in \mathbb{C} \backslash\{0\})$. The classes consisting of bi-starlike of Ma-Minda type of complex order $\gamma(\gamma \in \mathbb{C} \backslash\{0\})$ and bi-convex of Ma-Minda type of complex order $\gamma$ $(\gamma \in \mathbb{C} \backslash\{0\})$ are denoted by $\mathcal{S}_{\sigma}^{*}(\gamma ; \varphi)$ and $\mathcal{C}_{\sigma}(\gamma ; \varphi)$, respectively. As a special case $\gamma=1$ the classes $\mathcal{S}_{\sigma}^{*}(\gamma ; \varphi)$ and $\mathcal{C}_{\sigma}(\gamma ; \varphi)$ reduce to bi-starlike of Ma-Minda type and bi-convex of Ma-Minda type functions are denoted by $\mathcal{S}_{\sigma}^{*}(\varphi)$ and $\mathcal{C}_{\sigma}(\varphi)$, respectively.

In this paper, we consider more general class $\mathcal{S}_{\sigma}(\lambda, \gamma ; \varphi)$ for $0 \leq \lambda \leq 1, \gamma \in \mathbb{C} \backslash\{0\}$ which was investigated by Deniz [5] wherein he obtained the bounds for $a_{2}$ and $a_{3}$. This motivated us to study the Fekete-Szegö inequality to the class $\mathcal{S}_{\sigma}(\lambda, \gamma ; \varphi)$. Recently, some authors have investigated the Fekete-Szegö problem for various subclasses of $\sigma$ (see $[3,9,13,21,22])$.

\section{Coefficient estimates}

Throughout this paper $\varphi$ denotes an analytic univalent function in $\mathbb{D}$ with positive real part and normalized by $\varphi(0)=1, \varphi^{\prime}(0)>0$. Such a function has series expansion of the form

$$
\varphi(z)=1+B_{1} z+B_{2} z^{2}+B_{3} z^{3}+\ldots \quad\left(B_{1}>0\right) .
$$

Definition 2.1. For $0 \leq \lambda \leq 1$ and $\gamma \in \mathbb{C} \backslash\{0\}$, the class $\mathcal{S}(\lambda, \gamma ; \varphi)$ consists of functions $f \in \mathcal{A}$ satisfying

$$
1+\frac{1}{\gamma}\left(\frac{z f^{\prime}(z)+\lambda z^{2} f^{\prime \prime}(z)}{(1-\lambda) f(z)+\lambda z f^{\prime}(z)}-1\right) \prec \varphi(z) \quad(z \in \mathbb{D}) .
$$

The class $\mathcal{S}_{\sigma}(\lambda, \gamma ; \varphi)$ consists of functions $f \in \sigma$ such that $f, g \in \mathcal{S}(\lambda, \gamma ; \varphi)$ where $g$ is the analytic continuation of $f^{-1}$ to the unit disk $\mathbb{D}$.

The class $\mathcal{S}(\lambda, \gamma ; \varphi)$ was introduced by [18]. Motivated by this class the second author [5] defined and studied the class $\mathcal{S}_{\sigma}(\lambda, \gamma ; \varphi)$, which is called the class of generalized bi-subordinate functions of complex order $\gamma$ and type $\lambda$. As special cases of the class $\mathcal{S}_{\sigma}(\lambda, \gamma ; \varphi)$, we have $\mathcal{S}_{\sigma}(0, \gamma ; \varphi) \equiv \mathcal{S}_{\sigma}^{*}(\gamma ; \varphi)$ and $\mathcal{S}_{\sigma}(1, \gamma ; \varphi) \equiv \mathcal{C}_{\sigma}(\gamma ; \varphi)$.

The class $\mathcal{S}_{\sigma}(\lambda, \gamma ; \varphi)$ includes many earlier classes, which are mentioned below:

$\mathcal{S}_{\sigma}(0,1 ; \varphi) \equiv \mathcal{S}_{\sigma}^{*}(\varphi)$ and $\mathcal{S}_{\sigma}(1,1 ; \varphi) \equiv \mathcal{C}_{\sigma}(\varphi)$, are classes of Ma-Minda bi-starlike and MaMinda bi-convex functions, respectively, introduced and studied in [11].

$\mathcal{S}_{\sigma}\left((0,1 ;(1+A z) /(1+B z)) \equiv \mathcal{S}_{\sigma}[A, B]\right.$ and $\mathcal{S}_{\sigma}(1,1 ;(1+A z) /(1+B z)) \equiv \mathcal{C}_{\sigma}[A, B]$ $(-1 \leq B<A \leq 1)$ are, respectively, the classes of Janowski bi-starlike and bi-convex functions. Additionally, for $0 \leq \beta<1, \mathcal{S}_{\sigma}[1-2 \beta, 1] \equiv \mathcal{S}_{\sigma}(\beta)$ and $\mathcal{C}_{\sigma}[1-2 \beta, 1] \equiv \mathcal{C}_{\sigma}(\beta)$ are, respectively, the classes of bi-starlike and bi-convex functions of order $\beta$ introduced and studied in [2]. 
For $0<\beta \leq 1, \mathcal{S}_{\sigma}\left(0,1 ;\left(\frac{1+z}{1-z}\right)^{\beta}\right) \equiv \mathcal{S}_{\sigma}^{*}(\beta)$ and $\mathcal{S}_{\sigma}\left(1,1 ;\left(\frac{1+z}{1-z}\right)^{\beta}\right) \equiv \mathcal{S C}_{\sigma}^{*}(\beta)$ are, respectively, classes of strongly bi-starlike and strongly bi-convex functions of order $\beta$ introduced and studied in [2].

For $\gamma \in \mathbb{C} \backslash\{0\}, \mathcal{S}_{\sigma}(0, \gamma ;(1+z) /(1-z)) \equiv \mathcal{S}_{\sigma}^{*}[\gamma]$ and $\mathcal{S}_{\sigma}(1, \gamma ;(1+z) /(1-z)) \equiv \mathcal{C}_{\sigma}[\gamma]$ are classes of bi-starlike and bi-convex functions of complex order, respectively.

To prove our next theorems, we shall need the following well-known lemma (see [10]).

Lemma 2.2 ([10]). Let the function $w \in \mathcal{B}_{0}$ be given by

$$
w(z)=c_{1} z+c_{2} z^{2}+\cdots \quad(z \in \mathbb{D}),
$$

then for by every complex number $s$,

$$
\left|c_{2}-s c_{1}^{2}\right| \leq 1+(|s|-1)\left|c_{1}\right|^{2} .
$$

In the following theorem, we consider functional $\left|a_{3}-\mu a_{2}^{2}\right|$ for $\gamma$ nonzero complex number and $\mu \in \mathbb{C}$.

Theorem 2.3. Let the function $f$ given by (1.1) be in the $\mathcal{S}_{\sigma}(\lambda, \gamma ; \varphi)$. For $\gamma \in \mathbb{C} \backslash\{0\}$ and $\mu \in \mathbb{C}$, we have

$$
\begin{gathered}
\left|a_{2}\right| \leq \frac{|\gamma| B_{1}}{1+\lambda}, \\
\left|a_{3}\right| \leq \frac{|\gamma|\left|B_{1}\right|}{4(1+2 \lambda)} \max \{2,(|s|+|t|)\}
\end{gathered}
$$

and

$$
\left|a_{3}-\mu a_{2}^{2}\right| \leq \begin{cases}\frac{B_{1}|\gamma|}{2(1+2 \lambda)} & \text { if } \mathcal{L} \leq 2 \\ \frac{B_{1}|\gamma|}{4(1+2 \lambda)} \mathcal{L} & \text { if } \mathcal{L}>2\end{cases}
$$

where $s=\frac{B_{2}}{B_{1}}-\frac{4 B_{1} \gamma(1+2 \lambda)}{(1+\lambda)^{2}}, t=\frac{B_{2}}{B_{1}}$ and $\mathcal{L}=\left|\frac{B_{2}}{B_{1}}+(1-\mu) \frac{4 B_{1} \gamma(1+2 \lambda)}{(1+\lambda)^{2}}\right|+\left|\frac{B_{2}}{B_{1}}\right|$.

Proof. Since $f \in \mathcal{S}_{\sigma}(\lambda, \gamma ; \varphi)$, there exists two analytic functions $u, v: \mathbb{D} \rightarrow \mathbb{D}$, with $u(0)=0=v(0)$, such that

$$
1+\frac{1}{\gamma}\left(\frac{z f^{\prime}(z)+\lambda z^{2} f^{\prime \prime}(z)}{(1-\lambda) f(z)+\lambda z f^{\prime}(z)}-1\right)=\varphi(u(z)) \quad(z \in \mathbb{D})
$$

and

$$
1+\frac{1}{\gamma}\left(\frac{w g^{\prime}(w)+\lambda w^{2} g^{\prime \prime}(w)}{(1-\lambda) g(w)+\lambda w g^{\prime}(w)}-1\right)=\varphi(v(w)) .
$$

Define the functions $u$ and $v$ by

$$
u(z)=c_{1} z+c_{2} z^{2}+\cdots \text { and } v(w)=d_{1} w+d_{2} w^{2}+\cdots .
$$

Using (2.1) with (2.7), it is evident that

$$
\varphi(u(z))=1+\left(B_{1} c_{1}\right) z+\left(B_{1} c_{2}+B_{2} c_{1}^{2}\right) z^{2}+\cdots
$$

and

$$
\varphi(v(w))=1+\left(B_{1} d_{1}\right) w+\left(B_{1} d_{2}+B_{2} d_{1}^{2}\right) w^{2}+\cdots .
$$

Also, using (1.1), we get

$$
1+\frac{1}{\gamma}\left(\frac{z f^{\prime}(z)+\lambda z^{2} f^{\prime \prime}(z)}{(1-\lambda) f(z)+\lambda z f^{\prime}(z)}-1\right)=1+\frac{(1+\lambda) a_{2}}{\gamma} z+\left[\frac{2(1+2 \lambda) a_{3}-(1+\lambda)^{2} a_{2}^{2}}{\gamma}\right] z^{2}+\cdots
$$


and using (1.2), we get

$$
\begin{aligned}
1+ & \frac{1}{\gamma}\left(\frac{w g^{\prime}(w)+\lambda w^{2} g^{\prime \prime}(w)}{(1-\lambda) g(w)+\lambda w g^{\prime}(w)}-1\right) \\
& =1-\frac{(1+\lambda) a_{2}}{\gamma} w\left[\frac{-2(1+2 \lambda) a_{3}+\left(3+6 \lambda-\lambda^{2}\right) a_{2}^{2}}{\gamma}\right] w^{2}+\cdots
\end{aligned}
$$

Equating coefficients of right sides of equations (2.8) with (2.10) and (2.9) with (2.11) yield

$$
\frac{(1+\lambda) a_{2}}{\gamma}=B_{1} c_{1}, \quad \frac{2(1+2 \lambda) a_{3}-(1+\lambda)^{2} a_{2}^{2}}{\gamma}=B_{1} c_{2}+B_{2} c_{1}^{2}
$$

and

$$
\frac{-(1+\lambda) a_{2}}{\gamma}=B_{1} d_{1}, \quad \frac{-2(1+2 \lambda) a_{3}+\left(3+6 \lambda-\lambda^{2}\right) a_{2}^{2}}{\gamma}=B_{1} d_{2}+B_{2} d_{1}^{2}
$$

so that, on account of (2.12) and (2.13)

$$
\begin{aligned}
c_{1} & =-d_{1}, \\
a_{2} & =\frac{\gamma B_{1}}{1+\lambda} c_{1}
\end{aligned}
$$

and

$$
a_{3}=a_{2}^{2}+\frac{\gamma}{4(1+2 \lambda)}\left[B_{1} c_{2}+B_{2} c_{1}^{2}-B_{1} d_{2}-B_{2} d_{1}^{2}\right] .
$$

Taking into account (2.14), (2.15), (2.16) and the well known estimate $\left|c_{1}\right| \leq 1$ of the Schwarz lemma, we get

and from Lemma 2.2,

$$
\left|a_{2}\right|=\left|\frac{\gamma B_{1}}{1+\lambda} c_{1}\right| \leq \frac{|\gamma| B_{1}}{1+\lambda}
$$

$$
\begin{aligned}
\left|a_{3}\right| & =\left|a_{2}^{2}+\frac{\gamma}{4(1+2 \lambda)}\left[B_{1} c_{2}+B_{2} c_{1}^{2}-B_{1} d_{2}-B_{2} d_{1}^{2}\right]\right| \\
& =\left|\frac{\gamma^{2} B_{1}^{2}}{(1+\lambda)^{2}} c_{1}^{2}+\frac{\gamma}{4(1+2 \lambda)}\left[\left(B_{1} c_{2}-B_{2} c_{1}^{2}\right)-\left(B_{1} d_{2}-B_{2} d_{1}^{2}\right)\right]\right| \\
& =\left|\frac{\gamma B_{1}}{4(1+2 \lambda)}\left\{\left[c_{2}-\left(\frac{B_{2}}{B_{1}}-\frac{4 \gamma B_{1}(1+2 \lambda)}{(1+\lambda)^{2}}\right) c_{1}^{2}\right]-\left[d_{2}-\frac{B_{2}}{B_{1}} d_{1}^{2}\right]\right\}\right| \\
& \leq \frac{|\gamma| B_{1}}{4(1+2 \lambda)}\left\{\left|c_{2}-\left(\frac{B_{2}}{B_{1}}-\frac{4 \gamma B_{1}(1+2 \lambda)}{(1+\lambda)^{2}}\right) c_{1}^{2}\right|+\left|d_{2}-\frac{B_{2}}{B_{1}} d_{1}^{2}\right|\right\} \\
& \leq \frac{|\gamma| B_{1}}{4(1+2 \lambda)}\left\{1+(|s|-1)\left|c_{1}^{2}\right|+1+(|t|-1)\left|c_{1}^{2}\right|\right\} \\
& =\frac{|\gamma| B_{1}}{4(1+2 \lambda)}\left\{2+(|s|+|t|-2)\left|c_{1}^{2}\right|\right\} .
\end{aligned}
$$

Thus, using $\left|c_{1}\right| \leq 1$ we have the desired estimate for $\left|a_{3}\right|$ :

$$
\left|a_{3}\right| \leq \frac{|\gamma|\left|B_{1}\right|}{4(1+2 \lambda)} \max \{2,(|s|+|t|)\},
$$

where $s=\frac{B_{2}}{B_{1}}-\frac{4 B_{1} \gamma(1+2 \lambda)}{(1+\lambda)^{2}}$ and $t=\frac{B_{2}}{B_{1}}$.

To find an estimate for $\left|a_{3}-\mu a_{2}^{2}\right|$, we express $a_{3}-\mu a_{2}^{2}$ in terms of $c_{i}$ and $d_{i}$. Using the equality (2.16), we have

$$
a_{3}-\mu a_{2}^{2}=(1-\mu) a_{2}^{2}+\frac{\gamma}{4(1+2 \lambda)}\left[B_{1} c_{2}+B_{2} c_{1}^{2}-B_{1} d_{2}-B_{2} d_{1}^{2}\right] .
$$


Therefore from Lemma 2.2, we obtain

$$
\begin{aligned}
\left|a_{3}-\mu a_{2}^{2}\right| & =\left|(1-\mu) a_{2}^{2}+\frac{\gamma}{4(1+2 \lambda)}\left[B_{1} c_{2}+B_{2} c_{1}^{2}-B_{1} d_{2}-B_{2} d_{1}^{2}\right]\right| \\
& =\left|\frac{\gamma B_{1}}{4(1+2 \lambda)}\left\{\left[c_{2}-\left(\frac{B_{2}}{B_{1}}-(1-\mu) \frac{4 \gamma B_{1}(1+2 \lambda)}{(1+\lambda)^{2}}\right) c_{1}^{2}\right]-\left[d_{2}-\frac{B_{2}}{B_{1}} d_{1}^{2}\right]\right\}\right| \\
& \leq \frac{|\gamma| B_{1}}{4(1+2 \lambda)}\left\{2+\left(\left|\frac{B_{2}}{B_{1}}-(1-\mu) \frac{4 \gamma B_{1}(1+2 \lambda)}{(1+\lambda)^{2}}\right|+\left|\frac{B_{2}}{B_{1}}\right|-2\right)\left|c_{1}^{2}\right|\right\} \cdot(2.18)
\end{aligned}
$$

As a result of this, from $\left|c_{1}\right| \leq 1$ we obtain

$$
\left|a_{3}-\mu a_{2}^{2}\right| \leq \begin{cases}\frac{B_{1}|\gamma|}{2(1+2 \lambda)} & \text { if } \mathcal{L}<2 \\ \frac{B_{1}|\gamma|}{4(1+2 \lambda)} \mathcal{L} & \text { if } \mathcal{L} \geq 2\end{cases}
$$

where $\mathcal{L}=\left|\frac{B_{2}}{B_{1}}+(1-\mu) \frac{4 B_{1} \gamma(1+2 \lambda)}{(1+\lambda)^{2}}\right|+\left|\frac{B_{2}}{B_{1}}\right|$.

Thus the proof is completed.

We next consider the cases $\gamma$ and $\mu$ are real.

Theorem 2.4. Let the function $f$ given by (1.1) be in the $\mathcal{S}_{\sigma}(\lambda, \gamma ; \varphi)$. For $\gamma>0$ and $\mu \in \mathbb{R}$, we have

(1) If $\left|B_{2}\right| \geq B_{1}$, then

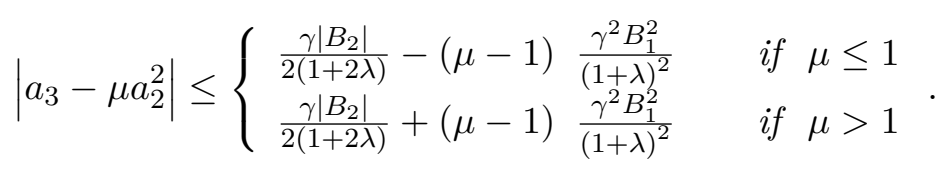

(2) If $\left|B_{2}\right|<B_{1}$, then

$$
\left|a_{3}-\mu a_{2}^{2}\right| \leq\left\{\begin{array}{lll}
\frac{\gamma\left|B_{2}\right|}{2(1+2 \lambda)}-(\mu-1) & \frac{\gamma^{2} B_{1}^{2}}{(1+\lambda)^{2}} & \text { if } \mu \leq 1-\mathcal{F} \\
\frac{\gamma B_{1}}{2(1+2 \lambda)} & \text { if } 1-\mathcal{F}<\mu<1+\mathcal{F} \\
\frac{\gamma\left|B_{2}\right|}{2(1+2 \lambda)}+(\mu-1) \frac{\gamma^{2} B_{1}^{2}}{(1+\lambda)^{2}} & \text { if } \mu \geq 1+\mathcal{F}
\end{array}\right.
$$

where $\mathcal{F}=\frac{(1+\lambda)^{2}\left(B_{1}-\left|B_{2}\right|\right)}{2 \gamma B_{1}^{2}(1+2 \lambda)}$.

Proof. Using (2.18) and Lemma 2.2, we obtain

$$
\begin{aligned}
\left|a_{3}-\mu a_{2}^{2}\right| & =\left|\frac{\gamma B_{1}}{4(1+2 \lambda)}\left\{\left[c_{2}-\left(\frac{B_{2}}{B_{1}}-(1-\mu) \frac{4 \gamma B_{1}(1+2 \lambda)}{(1+\lambda)^{2}}\right) c_{1}^{2}\right]-\left[d_{2}-\frac{B_{2}}{B_{1}} d_{1}^{2}\right]\right\}\right| \\
& \leq \frac{\gamma B_{1}}{4(1+2 \lambda)}\left\{2+\left(\left|\frac{B_{2}}{B_{1}}-(1-\mu) \frac{4 \gamma B_{1}(1+2 \lambda)}{(1+\lambda)^{2}}\right|+\left|\frac{B_{2}}{B_{1}}\right|-2\right)\left|c_{1}^{2}\right|\right\} \\
& \leq \frac{\gamma B_{1}}{2(1+2 \lambda)}+\left\{\frac{\gamma\left(\left|B_{2}\right|-B_{1}\right)}{2(1+2 \lambda)}+|\mu-1| \frac{\gamma^{2} B_{1}^{2}}{(1+\lambda)^{2}}\right\}\left|c_{1}^{2}\right| .
\end{aligned}
$$

Now, the proof will be presented in two cases: 
Firstly, we consider the case $\left|B_{2}\right| \geq B_{1}$.

If $\mu \leq 1$, then using (2.19) and $\left|c_{1}\right| \leq 1$, we obtain

$$
\begin{aligned}
\left|a_{3}-\mu a_{2}^{2}\right| & \leq \frac{\gamma B_{1}}{2(1+2 \lambda)}+\left\{\frac{\gamma\left(\left|B_{2}\right|-B_{1}\right)}{2(1+2 \lambda)}+(1-\mu) \frac{\gamma^{2} B_{1}^{2}}{(1+\lambda)^{2}}\right\}\left|c_{1}^{2}\right| \\
& \leq \frac{\gamma B_{1}}{2(1+2 \lambda)}+\left\{\frac{\gamma\left(\left|B_{2}\right|-B_{1}\right)}{2(1+2 \lambda)}+(1-\mu) \frac{\gamma^{2} B_{1}^{2}}{(1+\lambda)^{2}}\right\} \\
& =\frac{\gamma\left|B_{2}\right|}{2(1+2 \lambda)}-(\mu-1) \frac{\gamma^{2} B_{1}^{2}}{(1+\lambda)^{2}} .
\end{aligned}
$$

If $\mu>1$, then using (2.19) and $\left|c_{1}\right| \leq 1$, we obtain

$$
\begin{aligned}
\left|a_{3}-\mu a_{2}^{2}\right| & \leq \frac{\gamma B_{1}}{2(1+2 \lambda)}+\left\{\frac{\gamma\left(\left|B_{2}\right|-B_{1}\right)}{2(1+2 \lambda)}+(\mu-1) \frac{\gamma^{2} B_{1}^{2}}{(1+\lambda)^{2}}\right\}\left|c_{1}^{2}\right| \\
& \leq \frac{\gamma B_{1}}{2(1+2 \lambda)}+\left\{\frac{\gamma\left(\left|B_{2}\right|-B_{1}\right)}{2(1+2 \lambda)}+(\mu-1) \frac{\gamma^{2} B_{1}^{2}}{(1+\lambda)^{2}}\right\} \\
& =\frac{\gamma\left|B_{2}\right|}{2(1+2 \lambda)}+(\mu-1) \frac{\gamma^{2} B_{1}^{2}}{(1+\lambda)^{2}} .
\end{aligned}
$$

Finally, we consider the case $\left|B_{2}\right|<B_{1}$. By using (2.19) and $\left|c_{1}\right| \leq 1$, we obtain the following results according to the cases of $\mu$ and $\mathcal{F}$.

For $\mu \leq 1-\mathcal{F}$, we have

$$
\begin{aligned}
\left|a_{3}-\mu a_{2}^{2}\right| & \leq \frac{\gamma B_{1}}{2(1+2 \lambda)}+\left\{\frac{\gamma\left(\left|B_{2}\right|-B_{1}\right)}{2(1+2 \lambda)}+(1-\mu) \frac{\gamma^{2} B_{1}^{2}}{(1+\lambda)^{2}}\right\}\left|c_{1}^{2}\right| \\
& \leq \frac{\gamma B_{1}}{2(1+2 \lambda)}+\left\{\frac{\gamma\left(\left|B_{2}\right|-B_{1}\right)}{2(1+2 \lambda)}+(1-\mu) \frac{\gamma^{2} B_{1}^{2}}{(1+\lambda)^{2}}\right\} \\
& =\frac{\gamma\left|B_{2}\right|}{2(1+2 \lambda)}-(\mu-1) \frac{\gamma^{2} B_{1}^{2}}{(1+\lambda)^{2}},
\end{aligned}
$$

and for $1-\mathcal{F}<\mu \leq 1$, we yield

$$
\begin{aligned}
\left|a_{3}-\mu a_{2}^{2}\right| & \leq \frac{\gamma B_{1}}{2(1+2 \lambda)}+\left\{\frac{\gamma\left(\left|B_{2}\right|-B_{1}\right)}{2(1+2 \lambda)}+(1-\mu) \frac{\gamma^{2} B_{1}^{2}}{(1+\lambda)^{2}}\right\}\left|c_{1}^{2}\right| \\
& \leq \frac{\gamma B_{1}}{2(1+2 \lambda)} .
\end{aligned}
$$

Similarly for $1<\mu<1+\mathcal{F}$, we obtain

$$
\begin{aligned}
\left|a_{3}-\mu a_{2}^{2}\right| & \leq \frac{\gamma B_{1}}{2(1+2 \lambda)}+\left\{\frac{\gamma\left(\left|B_{2}\right|-B_{1}\right)}{2(1+2 \lambda)}+(\mu-1) \frac{\gamma^{2} B_{1}^{2}}{(1+\lambda)^{2}}\right\}\left|c_{1}^{2}\right| \\
& \leq \frac{\gamma B_{1}}{2(1+2 \lambda)} .
\end{aligned}
$$

Finally for $\mu \geq 1+\mathcal{F}$, we have

$$
\begin{aligned}
\left|a_{3}-\mu a_{2}^{2}\right| & \leq \frac{\gamma B_{1}}{2(1+2 \lambda)}+\left\{\frac{\gamma\left(\left|B_{2}\right|-B_{1}\right)}{2(1+2 \lambda)}+(\mu-1) \frac{\gamma^{2} B_{1}^{2}}{(1+\lambda)^{2}}\right\}\left|c_{1}^{2}\right| \\
& \leq \frac{\gamma B_{1}}{2(1+2 \lambda)}+\left\{\frac{\gamma\left(\left|B_{2}\right|-B_{1}\right)}{2(1+2 \lambda)}+(\mu-1) \frac{\gamma^{2} B_{1}^{2}}{(1+\lambda)^{2}}\right\} \\
& =\frac{\gamma\left|B_{2}\right|}{2(1+2 \lambda)}+(\mu-1) \frac{\gamma^{2} B_{1}^{2}}{(1+\lambda)^{2}} .
\end{aligned}
$$


Thus the proof is completed.

Finally, we consider the cases of $\gamma$ nonzero complex number and $\mu \in \mathbb{R}$.

Theorem 2.5. Let the function $f$ given by (1.1) be in the $\mathcal{S}_{\sigma}(\lambda, \gamma ; \varphi)$. For $\gamma \in \mathbb{C} \backslash\{0\}$ and $\mu \in \mathbb{R}$, we have

$$
\begin{aligned}
& \text { (1) If } \frac{(1+|\sin \theta|)\left|B_{2}\right|}{2 B_{1}} \geq 1 \text {, then } \\
& \left|a_{3}-\mu a_{2}^{2}\right| \leq \begin{cases}\frac{|\gamma|^{2} B_{1}^{2}}{(1+\lambda)^{2}}\left(1-\mu-\Re\left(k_{1}\right)\right)+\frac{|\gamma|\left|B_{2}\right|(1+|\sin \theta|)}{4(1+2 \lambda)} & \text { if } \mu \leq 1-\Re\left(k_{1}\right) \\
\frac{|\gamma|\left|B_{2}\right|(1+|\sin \theta|)}{4(1+2 \lambda)}-\frac{|\gamma|^{2} B_{1}^{2}}{(1+\lambda)^{2}}\left(1-\mu-\Re\left(k_{1}\right)\right) & \text { if } \mu>1-\Re\left(k_{1}\right)\end{cases} \\
& \text { (2) If } \frac{(1+|\sin \theta|)\left|B_{2}\right|}{2 B_{1}}<1, \text { then } \\
& \left|a_{3}-\mu a_{2}^{2}\right| \leq\left\{\begin{array}{l}
\frac{|\gamma|^{2} B_{1}^{2}}{(1+\lambda)^{2}}\left(1-\mu-\Re\left(k_{1}\right)\right)+\frac{|\gamma|\left|B_{2}\right|(1+|\sin \theta|)}{4(1+2 \lambda)} \text { if } \mu \leq 1-\Re\left(k_{1}\right)+\mathcal{N} \\
\frac{|\gamma| B_{1}}{2(1+2 \lambda)} \\
\frac{|\gamma|\left|B_{2}\right|(1+|\sin \theta|)}{4(1+2 \lambda)}-\frac{|\gamma|^{2} B_{1}^{2}}{(1+\lambda)^{2}}\left(1-\mu-\Re\left(k_{1}\right)+\mathcal{N}<\mu<1-\Re\left(k_{1}\right)-\mathcal{N}\right.
\end{array}\right.
\end{aligned}
$$

where $k_{1}=\frac{B_{2}(1+\lambda)^{2} e^{i \theta}}{4 B_{1}^{2}|\gamma|(1+2 \lambda)},|\gamma|=\gamma e^{i \theta}$ and $\mathcal{N}=\frac{(1+\lambda)^{2}\left[\left|B_{2}\right|(1+|\sin \theta|)-2 B_{1}\right]}{4 B_{1}^{2}|\gamma|(1+2 \lambda)}$.

Proof. Let $f \in \mathcal{S}_{\sigma}(\lambda, \gamma ; \varphi)$. By using (2.18) and Lemma 2.2, then we obtain

$$
\begin{aligned}
\left|a_{3}-\mu a_{2}^{2}\right| \leq & \frac{|\gamma| B_{1}}{4(1+2 \lambda)}\left\{2+\left(\left|\frac{B_{2}}{B_{1}}-(1-\mu) \frac{4 \gamma B_{1}(1+2 \lambda)}{(1+\lambda)^{2}}\right|+\left|\frac{B_{2}}{B_{1}}\right|-2\right)\left|c_{1}^{2}\right|\right\} \\
= & \frac{|\gamma| B_{1}}{2(1+2 \lambda)}+\frac{|\gamma|^{2} B_{1}^{2}}{(1+\lambda)^{2}} \\
& \times\left[\left|(1-\mu)-\frac{B_{2}(1+\lambda)^{2}}{4 B_{1}^{2} \gamma(1+2 \lambda)}\right|+\frac{\left(\left|B_{2}\right|-2 B_{1}\right)(1+\lambda)^{2}}{4 B_{1}^{2}|\gamma|(1+2 \lambda)}\right]\left|c_{1}^{2}\right| .
\end{aligned}
$$

Taking $|\gamma|=\gamma e^{i \theta}, k_{1}=\frac{B_{2}(1+\lambda)^{2} e^{i \theta}}{4 B_{1}^{2}|\gamma|(1+2 \lambda)}$ and $l_{1}=\frac{\left(\left|B_{2}\right|-2 B_{1}\right)(1+\lambda)^{2}}{4 B_{1}^{2}|\gamma|(1+2 \lambda)}$, for $B_{1}, B_{2} \in \mathbb{R}$ and $B_{1}>0$, we rewrite

$$
\begin{aligned}
\left|a_{3}-\mu a_{2}^{2}\right| & \leq \frac{|\gamma| B_{1}}{2(1+2 \lambda)}+\frac{|\gamma|^{2} B_{1}^{2}}{(1+\lambda)^{2}}\left(\left|1-\mu-k_{1}\right|+l_{1}\right)\left|c_{1}^{2}\right| \\
& =\frac{|\gamma| B_{1}}{2(1+2 \lambda)}+\frac{|\gamma|^{2} B_{1}^{2}}{(1+\lambda)^{2}}\left(\left|1-\mu-\Re\left(k_{1}\right)-i\left(\operatorname{Im}\left(k_{1}\right) \mid+l_{1}\right)\right| c_{1}^{2} \mid\right. \\
& \leq \frac{|\gamma| B_{1}}{2(1+2 \lambda)}+\frac{|\gamma|^{2} B_{1}^{2}}{(1+\lambda)^{2}}\left(\left|1-\mu-\Re\left(k_{1}\right)\right|+\frac{\left|B_{2}\right|(1+\lambda)^{2}|\sin \theta|}{4 B_{1}^{2}|\gamma|(1+2 \lambda)}+l_{1}\right)\left|c_{1}^{2}\right| \\
& =\frac{|\gamma| B_{1}}{2(1+2 \lambda)}+\left[\frac{|\gamma|^{2} B_{1}^{2}}{(1+\lambda)^{2}}\left|1-\mu-\Re\left(k_{1}\right)\right|+\frac{|\gamma|\left[\left|B_{2}\right|(1+|\sin \theta|)-2 B_{1}\right]}{4(1+2 \lambda)}\right]\left|c_{1}^{2}\right| .
\end{aligned}
$$

Firstly, we consider the case $\frac{(1+|\sin \theta|)\left|B_{2}\right|}{2 B_{1} \mid} \geq 1$.

Let $\mu \leq 1-\Re\left(k_{1}\right)$. Then from $(2.20)$ and $\left|c_{1}\right| \leq 1$, we obtain

$$
\begin{aligned}
\left|a_{3}-\mu a_{2}^{2}\right| & \leq \frac{|\gamma| B_{1}}{2(1+2 \lambda)}+\left[\frac{|\gamma|^{2} B_{1}^{2}}{(1+\lambda)^{2}}\left|1-\mu-\Re\left(k_{1}\right)\right|+\frac{|\gamma|\left[\left|B_{2}\right|(1+|\sin \theta|)-2 B_{1}\right]}{4(1+2 \lambda)}\right]\left|c_{1}^{2}\right| \\
& \leq \frac{|\gamma| B_{1}}{2(1+2 \lambda)}+\frac{|\gamma|^{2} B_{1}^{2}}{(1+\lambda)^{2}}\left(1-\mu-\Re\left(k_{1}\right)\right)+\frac{|\gamma|\left[\left|B_{2}\right|(1+|\sin \theta|)-2 B_{1}\right]}{4(1+2 \lambda)} \\
& =\frac{|\gamma|^{2} B_{1}^{2}}{(1+\lambda)^{2}}\left(1-\mu-\Re\left(k_{1}\right)\right)+\frac{|\gamma|\left|B_{2}\right|(1+|\sin \theta|)}{4(1+2 \lambda)} .
\end{aligned}
$$


Let $\mu>1-\Re\left(k_{1}\right)$. Then from $(2.20)$ and $\left|c_{1}\right| \leq 1$, we yield

$$
\begin{aligned}
\left|a_{3}-\mu a_{2}^{2}\right| & \leq \frac{|\gamma| B_{1}}{2(1+2 \lambda)}+\left[\frac{|\gamma|^{2} B_{1}^{2}}{(1+\lambda)^{2}}\left|1-\mu-\Re\left(k_{1}\right)\right|+\frac{|\gamma|\left[\left|B_{2}\right|(1+|\sin \theta|)-2 B_{1}\right]}{4(1+2 \lambda)}\right]\left|c_{1}^{2}\right| \\
& \leq \frac{|\gamma| B_{1}}{2(1+2 \lambda)}+\frac{|\gamma|^{2} B_{1}^{2}}{(1+\lambda)^{2}}\left(\mu+\Re\left(k_{1}\right)-1\right)+\frac{|\gamma|\left[\left|B_{2}\right|(1+|\sin \theta|)-2 B_{1}\right]}{4(1+2 \lambda)} \\
& =\frac{|\gamma|\left|B_{2}\right|(1+|\sin \theta|)}{4(1+2 \lambda)}-\frac{|\gamma|^{2} B_{1}^{2}}{(1+\lambda)^{2}}\left(1-\mu-\Re\left(k_{1}\right)\right) .
\end{aligned}
$$

Finally, we want to consider the case with $\frac{(1+|\sin \theta|)\left|B_{2}\right|}{2 B_{1}}<1$. By using $(2.20)$ and $\left|c_{1}\right| \leq 1$, we obtain the following results according to the cases of $\mu, k_{1}$ and $\mathcal{N}$.

For $\mu \leq 1-\Re\left(k_{1}\right)+\mathcal{N}$, we have

$$
\begin{aligned}
\left|a_{3}-\mu a_{2}^{2}\right| & \leq \frac{|\gamma| B_{1}}{2(1+2 \lambda)}+\left[\frac{|\gamma|^{2} B_{1}^{2}}{(1+\lambda)^{2}}\left|1-\mu-\Re\left(k_{1}\right)\right|+\frac{|\gamma|\left[\left|B_{2}\right|(1+|\sin \theta|)-2 B_{1}\right]}{4(1+2 \lambda)}\right]\left|c_{1}^{2}\right| \\
& \leq \frac{|\gamma| B_{1}}{2(1+2 \lambda)}+\frac{|\gamma|^{2} B_{1}^{2}}{(1+\lambda)^{2}}\left(1-\mu-\Re\left(k_{1}\right)\right)+\frac{|\gamma|\left[\left|B_{2}\right|(1+|\sin \theta|)-2 B_{1}\right]}{4(1+2 \lambda)} \\
& =\frac{|\gamma|^{2} B_{1}^{2}}{(1+\lambda)^{2}}\left(1-\mu-\Re\left(k_{1}\right)\right)+\frac{|\gamma|\left|B_{2}\right|(1+|\sin \theta|)}{4(1+2 \lambda)},
\end{aligned}
$$

and for $1-\Re\left(k_{1}\right)+\mathcal{N}<\mu \leq 1-\Re\left(k_{1}\right)$, we obtain

$$
\begin{aligned}
\left|a_{3}-\mu a_{2}^{2}\right| & \leq \frac{|\gamma| B_{1}}{2(1+2 \lambda)}+\left[\frac{|\gamma|^{2} B_{1}^{2}}{(1+\lambda)^{2}}\left|1-\mu-\Re\left(k_{1}\right)\right|+\frac{|\gamma|\left[\left|B_{2}\right|(1+|\sin \theta|)-2 B_{1}\right]}{4(1+2 \lambda)}\right]\left|c_{1}^{2}\right| \\
& \leq \frac{|\gamma| B_{1}}{2(1+2 \lambda)} .
\end{aligned}
$$

Similarly, for $1-\Re\left(k_{1}\right)<\mu<1-\Re\left(k_{1}\right)-\mathcal{N}$, we yield

$$
\begin{aligned}
\left|a_{3}-\mu a_{2}^{2}\right| & \leq \frac{|\gamma| B_{1}}{2(1+2 \lambda)}+\left[\frac{|\gamma|^{2} B_{1}^{2}}{(1+\lambda)^{2}}\left|1-\mu-\Re\left(k_{1}\right)\right|+\frac{|\gamma|\left[\left|B_{2}\right|(1+|\sin \theta|)-2 B_{1}\right]}{4(1+2 \lambda)}\right]\left|c_{1}^{2}\right| \\
& \leq \frac{|\gamma| B_{1}}{2(1+2 \lambda)},
\end{aligned}
$$

and finally, for $\mu \geq 1-\Re\left(k_{1}\right)-\mathcal{N}$, we have

$$
\begin{aligned}
\left|a_{3}-\mu a_{2}^{2}\right| & \leq \frac{|\gamma| B_{1}}{2(1+2 \lambda)}+\left[\frac{|\gamma|^{2} B_{1}^{2}}{(1+\lambda)^{2}}\left|1-\mu-\Re\left(k_{1}\right)\right|+\frac{|\gamma|\left[\left|B_{2}\right|(1+|\sin \theta|)-2 B_{1}\right]}{4(1+2 \lambda)}\right]\left|c_{1}^{2}\right| \\
& \leq \frac{|\gamma| B_{1}}{2(1+2 \lambda)}+\frac{|\gamma|^{2} B_{1}^{2}}{(1+\lambda)^{2}}\left(\mu+\Re\left(k_{1}\right)-1\right)+\frac{|\gamma|\left[\left|B_{2}\right|(1+|\sin \theta|)-2 B_{1}\right]}{4(1+2 \lambda)} \\
& =\frac{|\gamma|\left|B_{2}\right|(1+|\sin \theta|)}{4(1+2 \lambda)}-\frac{|\gamma|^{2} B_{1}^{2}}{(1+\lambda)^{2}}\left(1-\mu-\Re\left(k_{1}\right)\right) .
\end{aligned}
$$

Thus the proof is completed.

Taking $\gamma=1, \lambda=0$ and $\varphi(z)=(1+A z) /(1+B z) \quad(-1 \leq B<A \leq 1)$ in Theorems $2.3,2.4$ and 2.5 , we have the following corollary.

Corollary 2.6. If $f \in \mathcal{A}$ is given by (1.1) belongs to the class $\mathcal{S}_{\sigma}[A, B]$, then

(1) For $\mu \in \mathbb{C}$,

$$
\left|a_{3}-\mu a_{2}^{2}\right| \leq\left\{\begin{array}{ll}
\frac{A-B}{2} & \text { if }|B|+|4(1-\mu)(A-B)-B|<2 \\
\frac{(A-B)}{4}[|B|+|4(1-\mu)(A-B)-B|] & \text { if }|B|+|4(1-\mu)(A-B)-B| \geq 2
\end{array} .\right.
$$


(2) For $\mu \in \mathbb{R}$,

$$
\left|a_{3}-\mu a_{2}^{2}\right| \leq\left\{\begin{array}{lll}
\frac{|B|(A-B)}{2}-(\mu-1) & (A-B)^{2} & \text { if } \quad \mu \leq 1-\frac{1-|B|}{2(A-B)} \\
\frac{A-B}{2} & \text { if } 1-\frac{1-|B|}{2(A-B)}<\mu<1+\frac{1-|B|}{2(A-B)} \\
\frac{|B|(A-B)}{2}+(\mu-1) \quad(A-B)^{2} & \text { if } \mu \geq 1+\frac{1-|B|}{2(A-B)}
\end{array}\right.
$$

and

$$
\left|a_{3}-\mu a_{2}^{2}\right| \leq\left\{\begin{array}{lll}
(A-B) & {\left[(A-B)(1-\mu)+\frac{|B|+B}{4}\right]} & \text { if } \mu \leq 1+\frac{|B|+B-2}{4(A-B)} \\
\frac{A-B}{2} & \text { if } 1+\frac{|B|+B-2}{4(A-B)}<\mu<1-\frac{|B|-B-2}{4(A-B)} \\
(A-B) & {\left[(A-B)(\mu-1)+\frac{|B|-B}{4}\right]} & \text { if } \mu \geq 1-\frac{|B|-B-2}{4(A-B)}
\end{array} .\right.
$$

Taking $\gamma=1, \lambda=1$ and $\varphi(z)=(1+A z) /(1+B z) \quad(-1 \leq B<A \leq 1)$ in Theorems 2.3, 2.4 and 2.5 , we have the following corollary.

Corollary 2.7. If $f \in \mathcal{A}$ is given by (1.1) belongs to the class $\mathcal{C}_{\sigma}[A, B]$, then

(1) For $\mu \in \mathbb{C}$,

$$
\left|a_{3}-\mu a_{2}^{2}\right| \leq\left\{\begin{array}{lr}
\frac{A-B}{6} & \text { if }|B|+|3(1-\mu)(A-B)-B|<2 \\
\frac{(A-B)}{12}[|B|+|3(1-\mu)(A-B)-B|] & \text { if }|B|+|3(1-\mu)(A-B)-B| \geq 2
\end{array}\right. \text {. }
$$

(2) For $\mu \in \mathbb{R}$,

$$
\begin{aligned}
& \left|a_{3}-\mu a_{2}^{2}\right| \leq \begin{cases}\frac{|B|(A-B)}{6}-(\mu-1) \frac{(A-B)^{2}}{4} & \text { if } \mu \leq 1-\frac{2(1-|B|)}{3(A-B)} \\
\frac{A-B}{6} & \text { if } 1-\frac{2(1-|B|)}{3(A-B)}<\mu<1+\frac{2(1-|B|)}{3(A-B)} \\
\frac{|B|(A-B)}{6}+(\mu-1) \frac{(A-B)^{2}}{4} & \text { if } \mu \geq 1+\frac{2(1-|B|)}{3(A-B)}\end{cases} \\
& \quad \text { and } \quad \text { if } \mu \leq 1+\frac{2|B|+2 B-1}{6(A-B)} \\
& \left|a_{3}-\mu a_{2}^{2}\right| \leq \begin{cases}\frac{A-B}{12}[3(A-B)(1-\mu)+|B|+B] \\
\frac{A-B}{6} & \text { if } 1+\frac{2|B|+2 B-1}{6(A-B)}<\mu<1-\frac{2|B|-2 B-1}{6(A-B)} \\
\frac{A-B}{12}[3(A-B)(\mu-1)+|B|-B] & \text { if } \mu \geq 1-\frac{2|B|-2 B-1}{6(A-B)}\end{cases}
\end{aligned}
$$

Taking $\gamma \in \mathbb{C} \backslash\{0\}, \lambda=0$ and $\varphi(z)=(1+z) /(1-z)$ in Theorems 2.3, 2.4 and 2.5, then we have the following corollary.

Corollary 2.8. If $f \in \mathcal{A}$ is given by (1.1) belongs to the class $\mathcal{S}_{\sigma}^{*}[\gamma]$, then

(i) For $\gamma \in \mathbb{C} \backslash\{0\}$ and $\mu \in \mathbb{C}$,

$$
\left|a_{3}-\mu a_{2}^{2}\right| \leq\left\{\begin{array}{ll}
|\gamma| & \text { if } \quad|1+(1-\mu) 8 \gamma|<1 \\
\frac{|\gamma|}{2}[|1+(1-\mu) 8 \gamma|+1] & \text { if } \quad|1+(1-\mu) 8 \gamma| \geq 1
\end{array} .\right.
$$

(ii) For $\gamma>0$ and $\mu \in \mathbb{R}$,

$$
\left|a_{3}-\mu a_{2}^{2}\right| \leq\left\{\begin{array}{ll}
\gamma-4(\mu-1) \gamma^{2} & \text { if } \quad \mu \leq 1 \\
\gamma+4(\mu-1) \gamma^{2} & \text { if } \quad \mu>1
\end{array} .\right.
$$

(iii) For $\gamma \in \mathbb{C} \backslash\{0\}$ and $\mu \in \mathbb{R}$,

$$
\begin{gathered}
\left|a_{3}-\mu a_{2}^{2}\right| \leq\left\{\begin{array}{lc}
4|\gamma|^{2}(1-\mu)+\frac{|\gamma|(1+|\sin \theta|-\cos \theta)}{2} & \text { if } \mu \leq 1+\chi_{1}(\gamma, \theta) \\
|\gamma| & \text { if } 1+\chi_{1}(\gamma, \theta)<\mu<1-\chi_{2}(\gamma, \theta) \\
\frac{|\gamma|(1+|\sin \theta|-\cos \theta)}{2}-4|\gamma|^{2}(1-\mu) & \text { if } \mu \geq 1-\chi_{2}(\gamma, \theta)
\end{array}\right. \\
\text { where } \chi_{1}(\gamma, \theta)=\frac{(|\sin \theta|-\cos \theta-1)}{8|\gamma|} \text { and } \chi_{2}(\gamma, \theta)=\frac{(|\sin \theta|+\cos \theta-1)}{8|\gamma|} .
\end{gathered}
$$


Taking $\gamma \in \mathbb{C} \backslash\{0\}, \lambda=1$ and $\varphi(z)=(1+z) /(1-z)$ in Theorems 2.3, 2.4 and 2.5, we obtain the following corollary.

Corollary 2.9. If $f \in \mathcal{A}$ is given by (1.1) belongs to the class $\mathcal{C}_{\sigma}[\gamma]$, then

(i) For $\gamma \in \mathbb{C} \backslash\{0\}$ and $\mu \in \mathbb{C}$,

$$
\left|a_{3}-\mu a_{2}^{2}\right| \leq\left\{\begin{array}{ll}
\frac{|\gamma|}{3} & \text { if }|1+(1-\mu) 6 \gamma|<1 \\
\frac{|\gamma|}{2}[|1+(1-\mu) 6 \gamma|+1] & \text { if }|1+(1-\mu) 6 \gamma| \geq 1
\end{array} .\right.
$$

(ii) For $\gamma>0$ and $\mu \in \mathbb{R}$,

$$
\left|a_{3}-\mu a_{2}^{2}\right| \leq\left\{\begin{array}{ll}
\frac{\gamma}{3}-(\mu-1) \gamma^{2} & \text { if } \mu \leq 1 \\
\frac{\gamma}{3}+(\mu-1) \gamma^{2} & \text { if } \mu>1
\end{array} .\right.
$$

(iii) For $\gamma \in \mathbb{C} \backslash\{0\}$ and $\mu \in \mathbb{R}$,

$$
\begin{gathered}
\left|a_{3}-\mu a_{2}^{2}\right| \leq \begin{cases}|\gamma|^{2}(1-\mu)+\frac{|\gamma|(1+|\sin \theta|-\cos \theta)}{6} & \text { if } \mu \leq 1+\varphi_{1}(\gamma, \theta) \\
\frac{|\gamma|}{3} & \text { if } 1+\varphi_{1}(\gamma, \theta)<\mu<1-\varphi_{2}(\gamma, \theta) \\
\frac{|\gamma|(1+|\sin \theta|-\cos \theta)}{6}-|\gamma|^{2}(1-\mu) & \text { if } \mu \geq 1-\varphi_{2}(\gamma, \theta)\end{cases} \\
\text { where } \varphi_{1}(\gamma, \theta)=\frac{(|\sin \theta|-\cos \theta-1)}{6|\gamma|} \text { and } \varphi_{2}(\gamma, \theta)=\frac{(|\sin \theta|+\cos \theta-1)}{6|\gamma|} .
\end{gathered}
$$

Acknowledgment. The research of E. Deniz was supported by the Commission for the Scientific Research Projects of Kafkas University, project number 2016-FM-67.

\section{References}

[1] R.M. Ali, S.K. Lee, V. Ravichandran, and S. Supramaniam, Coefficient estimates for bi-univalent Ma-Minda starlike and convex functions, Appl. Math. Lett. 25, 344-351, 2012.

[2] D.A. Brannan, and T.S. Taha, On some classes of bi-univalent functions, Studia Univ. Babe-Bolyai Math. 31, 70-77, 1986.

[3] S. Bulut, Coefficient estimates for a subclass of parabolic bi-starlike functions, Afr. Math. 29, 331-338, 2018.

[4] M. Çă̆lar, H. Orhan and N. Yağmur, Coefficient bounds for new subclasses of biunivalent functions, Filomat 27, 1165-1171, 2013.

[5] E. Deniz, Certain subclasses of bi-univalent functions satisfying subordinate conditions, J. Class. Anal. 2, 49-60, 2013.

[6] B.A. Frasin and M.K. Aouf, New subclasses of bi-univalent functions, Appl. Math. Lett. 24, 1569-1573, 2011.

[7] J.M. Jahangiri and S.G. Hamidi, Coefficient estimates for certain classes of biunivalent functions, Int. J. Math. Math. Sci. Article ID 190560, 4 pp. 2013.

[8] J.M. Jahangiri, S.G. Hamidi and S.A. Halim, Coefficients of bi-univalent functions with positive real part derivatives, Bull. Malay. Math. Sci. Soc. 37, 633-640, 2014.

[9] J.M. Jahangiri, N. Magesh and J. Yamini, Fekete-Szegö inequalities for classes of bi-starlike and bi-convex functions, Electron. J. Math. Anal. Appl. 3, 133-140, 2015.

[10] F.R. Keogh and E.P. Merkes, A coefficient inequality for certain classes of analytic functions, Proc. Amer. Math. Soc. 20, 8-12, 1969.

[11] S.S. Kumar, V. Kumar and V. Ravichandran, Estimates for the initial coefficients of bi-univalent functions, Tamsui Oxford J. Inform. Math. Sci. 29, 487-504, 2013.

[12] W.C. Ma and D. Minda, A unified treatment of some special classes of univalent functions, Proceedings of the Conference on Complex Analysis, Tianjin, 1992; Conf. Proc. Lecture. Notes Anal. I, Int Press, Cambridge, MA, 157-169, 1994.

[13] H. Orhan, N. Magesh and V.K. Balaji, Fekete-Szegö problem for certain classes of Ma-Minda bi-univalent functions, Afr. Math. 27, 889-897, 2016. 
[14] V. Ravichandran, Y. Polatoğlu, M. Bolcal and A. Şen, Certain subclasses of starlike and convex functions of complex order, Hacettepe J. Math. Stat. 34, 9-15, 2005.

[15] H.M. Srivastava, S. Bulut, M. Çă̆lar and N. Yağmur, Coefficient estimates for a general subclass of analytic and bi-univalent functions, Filomat 27, 831-842, 2013.

[16] H.M. Srivastava, S.S Eker, and R.M. Ali, Coefficient bounds for a certain class of analytic and bi-univalent functions, Filomat 29, 1839-1845, 2015.

[17] H.M. Srivastava, A.K. Mishra, and P. Gochhayat, Certain subclasses of analytic and bi-univalent functions, Appl. Math. Lett. 23, 1188-1192, 2010.

[18] Q.H. Xu, Y.C. Gui and H.M. Srivastava, Coefficient estimates for certain subclasses of analytic functions of complex order, Taiwanese J. Math. 15, 2377-2386, 2011.

[19] Q.H. Xu, Y.C. Gui and H.M. Srivastava, Coefficient estimates for a certain subclass of analytic and bi-univalent functions, Appl. Math. Lett. 25, 990-994, 2012.

[20] Q.H. Xu, H.G. Xiao and H.M Srivastava, A certain general subclass of analytic and bi-univalent functions and associated coefficient estimate problems, Appl. Math. Comput. 218, 11461-11465, 2012.

[21] P. Zaprawa, Estimates of Initial Coefficients for Bi-Univalent Functions, Abst. Appl. Anal. Article ID 357480, 6 pp. 2014.

[22] P. Zaprawa, On the Fekete-Szegö problem for classes of bi-univalent functions, Bull. Belg. Math. Soc. Simon Stevin 21, 169-178, 2014. 